\title{
Saturation Numbers for Trees
}

\author{
Jill Faudree \\ Department of Mathematics and Statistics \\ University of Alaska Fairbanks \\ Fairbanks, AK 99775-6660 \\ ffjrf@uaf.edu \\ Ronald J. Gould \\ Department of Mathematics and Computer Science \\ Emory University \\ Atlanta, GA 30322 \\ rg@mathcs . emory . edu
}

\author{
Ralph J. Faudree \\ Department of Mathematical Sciences \\ University of Memphis \\ Memphis, TN 38152 \\ rfaudree@memphis.edu \\ Michael S. Jacobson \\ Department of Mathematics \\ University of Colorado Denver \\ Denver, CO 80217 \\ msj@math . cudenver . edu
}

Submitted: Jan 30, 2009; Accepted: Jul 17 2009; Published: Jul 24, 2009

Mathematics Subject Classifications: 05C35, 05C05

\begin{abstract}
For a fixed graph $H$, a graph $G$ is $H$-saturated if there is no copy of $H$ in $G$, but for any edge $e \notin G$, there is a copy of $H$ in $G+e$. The collection of $H$ saturated graphs of order $n$ is denoted by $\mathbf{S A T}(n, H)$, and the saturation number, $\operatorname{sat}(n, H)$, is the minimum number of edges in a graph in $\operatorname{SAT}(n, H)$. Let $T_{k}$ be a tree on $k$ vertices. The saturation numbers $\operatorname{sat}\left(n, T_{k}\right)$ for some families of trees will be determined precisely. Some classes of trees for which $\operatorname{sat}\left(n, T_{k}\right)<n$ will be identified, and trees $T_{k}$ in which graphs in $\mathbf{S A T}\left(n, T_{k}\right)$ are forests will be presented. Also, families of trees for which $\operatorname{sat}\left(n, T_{k}\right) \geq n$ will be presented. The maximum and minimum values of $\operatorname{sat}\left(n, T_{k}\right)$ for the class of all trees will be given. Some properties of $\mathbf{s a t}\left(n, T_{k}\right)$ and $\mathbf{S A T}\left(n, T_{k}\right)$ for trees will be discussed.
\end{abstract}

\section{Introduction and Notation}

Only finite graphs without loops or multiple edges will be considered. Notation will be standard, and generally follow the notation of [CL05]. For a graph $G$ we use $G$ to represent the vertex set $V(G)$ and the edge set $E(G)$ when it is clear from the context.

For a fixed graph $H$, a graph $G$ is $H$-saturated if there is no copy of $H$ in $G$, but for any edge $e \notin G$, there is a copy of $H$ in $G+e$. The collection of $H$-saturated graphs of order $n$ is denoted by $\operatorname{SAT}(n, H)$, and the saturation number, denoted $\operatorname{sat}(n, H)$, is the minimum number of edges in a graph in $\operatorname{SAT}(n, H)$. The maximum number of edges in a graph in $\operatorname{SAT}(n, H)$ is the well known Turán extremal number (see [Tur41]), and 
is usually denoted by $\operatorname{ex}(n, H)$. The graphs in $\operatorname{SAT}(n, H)$ with a minimum number of edges will be denoted by $\underline{\mathbf{S A T}}(n, H)$, and those with a maximum number of edges will be denoted by $\overline{\mathbf{S A T}}(n, H)$. Thus, all graphs in $\underline{\mathbf{S A T}}(n, H)$ have $\boldsymbol{\operatorname { s a t }}(n, H)$ edges and graphs in $\overline{\mathbf{S A T}}(n, H)$ have $e x(n, H)$ edges.

We will denote a path on $k$ vertices by $P_{k}$. The complete bipartite graph $K_{1, k-1}$ be be called a star (on $k$ vertices) and will be denoted by $S_{k}$. The vertex of degree $k-1$ is called the center of the star. A double star, denoted $S_{t, r}$, is the graph on $t+r$ vertices constructed by adding an edge between the centers of a star on $t$ vertices and a star on $r$ vertices. (See Figure 1.) When $t=r$, we say $S_{t, t}$ is a symmetric double star.

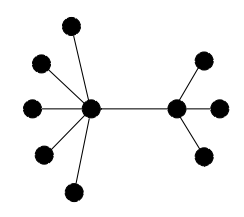

Figure 1: $S_{6,4}$

The notion of the saturation number of a graph was introduced by Erdős, Hajnal, and Moon in [EHM64] in which the authors proved $\operatorname{sat}\left(n, K_{t}\right)=\left(\begin{array}{c}t-2 \\ 2\end{array}\right)+(n-t+2)(t-2)$ and $\underline{\operatorname{SAT}}\left(n, K_{t}\right)=\left\{K_{t-2}+\bar{K}_{n-t+2}\right\}$. Since then $\operatorname{sat}(n, G)$ and $\underline{\operatorname{SAT}}(n, G)$ have been investigated for a range of graphs $G$. Some additional examples of graphs for which the saturation number is known precisely include small cycles [Oll72] [Che09], complete bipartite graphs [Bol67], matchings [KT86], and books [CFG08]. The exact value of $\operatorname{sat}(n, G)$ and a complete characterization of $\underline{\operatorname{SAT}}(n, G)$ are known for very few graphs $G$. For a summary of known results see [GGL95] Chapter 23 or [FFS09]. Generalizations to hypergraphs also exist, see [Pik04].

The emphasis of this paper will be on exploring $\operatorname{sat}\left(n, T_{k}\right)$ when the graph $T_{k}$ is a tree of order $k$. For special trees, specifically paths and stars, the saturation numbers are already known. These results will be discussed in Section 2. The saturation numbers $\operatorname{sat}\left(n, T_{k}\right)$ for some families of trees will be determined precisely such as when $T_{k}$ is a broom, double star, and subdivided star. The class of trees for which $\operatorname{sat}\left(n, T_{k}\right)<n$ will be explored, and large classes of trees will be shown to have this property. Such trees are said to have "small" saturation numbers, and these are trees $T_{k}$ in which some of the graphs in $\operatorname{SAT}\left(n, T_{k}\right)$ are forests. Also, families of trees in which $\operatorname{sat}\left(n, T_{k}\right) \geq n$ will be studied. The minimum values of $\operatorname{sat}\left(n, T_{k}\right)$ for the class of all trees on $k$ vertices will be determined. Some properties of $\operatorname{sat}\left(n, T_{k}\right)$ and $\mathbf{S A T}\left(n, T_{k}\right)$ for trees will be discussed.

\section{Known Results}

In [KT86] Kászonyi and Tuza proved several general results concerning saturated graphs including an upper bound for $\operatorname{sat}(n, H)$ for any connected graph $H$ by constructing an $H$-saturated graph. It should be noted that Z. Furedi [Für09] has recently presented 
an alternate and somewhat shorter proof of this upper bound. The results particularly relevant here are those concerning stars and paths which are summarized below.

Theorem 1. [KT86] Saturation Numbers for Paths and Stars

(a) $\operatorname{sat}\left(n, S_{k+1}\right)= \begin{cases}\left(\begin{array}{c}k \\ 2\end{array}\right)+\left(\begin{array}{c}n-k \\ 2\end{array}\right) & \text { if } k+1 \leq n \leq k+\frac{k}{2} \\ \left\lceil\frac{k-1}{2} n-\frac{k^{2}}{8}\right\rceil & \text { if } k+\frac{k}{2} \leq n\end{cases}$

(b) For $n \geq 3$, $\operatorname{sat}\left(n, P_{3}\right)=\lfloor n / 2\rfloor$.

(c) For $n \geq 4, \operatorname{sat}\left(n, P_{4}\right)= \begin{cases}n / 2 & n \text { even }, \\ (n+3) / 2 & n \text { odd. }\end{cases}$

(d) For $n \geq 5$, $\operatorname{sat}\left(n, P_{5}\right)=\left\lceil\frac{5 n-4}{6}\right\rceil$.

(e) Let $a_{k}=\left\{\begin{array}{ll}3 \cdot 2^{t-1}-2 & \text { if } k=2 t, \\ 4 \cdot 2^{t-1}-2 & \text { if } k=2 t+1 .\end{array}\right.$ If $n \geq a_{k}$ and $k \geq 6$, then $\operatorname{sat}\left(n, P_{k}\right)=$ $n-\left\lfloor\frac{n}{a_{k}}\right\rfloor$.

Theorem 2. [KT86] The Set of Minimal Star-Saturated Graphs

$$
\underline{\operatorname{SAT}}\left(n, S_{k}\right)= \begin{cases}K_{k-1} \cup K_{n-k+1} & \text { if } k \leq n \leq \frac{3 k-3}{2}, \\ G^{\prime} \cup K_{p} & \text { if } \frac{3 k-3}{2} \leq n,\end{cases}
$$

where $p=\lfloor k / 2\rfloor$ and $G^{\prime}$ is a $(k-1)$-regular graph on $n-p$ vertices. Note that in the case when $n \geq \frac{3 k-3}{2}$, an edge is added if $k-1$ and $n-p$ are both odd.

The set $\underline{\operatorname{SAT}}\left(n, P_{k}\right)$ is more complicated and may contain many nonisomorphic graphs. However, all minimal $P_{k}$-saturated trees do have a common structure which will be useful later. Thus, we make the following definitions. In these definitions, $l$ and $d$ are integers.

A perfect $d$-ary tree is a tree such that every vertex has degree $d$ or degree 1 and all degree 1 vertices are the same distance from the center. Thus for a given $d$, a pair of distinct perfect $d$-ary trees differ by their diameter. Hence, we let $T_{l, d}$ denote the perfect $d$-ary tree whose longest path contains $l$ vertices (i.e. $T_{l, d}$ has diameter $l-1$ ). (See Figure 2.)
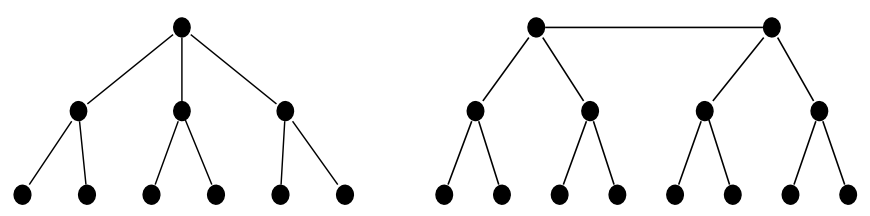

Figure 2: $T_{5,3}$ and $T_{6,3}$

In some instances it will be useful to view $T_{l, d}$ as a rooted (or double rooted) tree. Specifically, if $l$ is odd, let the root $r$ be the unique vertex in the center of $T_{l, d}$. Viewed 
in this way, the tree has $\left\lceil\frac{l}{2}\right\rceil$ levels, the root has $d$ children, all vertices in the middle levels have $d-1$ children, all vertices of degree 1 are in the bottom level, and $\left|V\left(T_{l, d}\right)\right|=$ $\frac{d(d-1)^{\lfloor l / 2\rfloor}-2}{d-2}$. If $l$ is even, the center consists of two adjacent vertices which we call $r_{1}$ and $r_{2}$ and we consider these to be the roots of the tree. In this case, all vertices have $d-1$ children except for those of degree 1 all of which are in the bottom of the $l / 2$ levels and $\left|V\left(T_{l, d}\right)\right|=2\left(\frac{(d-1)^{l / 2}-2}{d-2}\right)$.

Observe that $T_{l-1, d}$ is $P_{l}$-saturated for all $d \geq 3$. In addition, any graph obtained from $T_{l-1, d}$ by adding more pendant vertices to those already adjacent to vertices of degree 1 maintains the $P_{l}$-saturated property. In the theorem below, observe that $a_{l}=\left|V\left(T_{l-1,3}\right)\right|$.

Theorem 3. [KT86] The Set of Minimal Path-Saturated Graphs

Let $P_{k}$ be a path on $k \geq 3$ vertices and let $T_{k-1,3}$ be the tree defined above.

Let $a_{k}=\left\{\begin{array}{ll}3 \cdot 2^{m-1}-2 & \text { if } k=2 m, \\ 4 \cdot 2^{m-1}-2 & \text { if } k=2 m+1 .\end{array}\right.$ Then, for $n \geq a_{k}, \underline{\operatorname{SAT}}\left(n, P_{k}\right)$ consists of a forest with $\left\lfloor n / a_{k}\right\rfloor$ components. Furthermore, if $T$ is a $P_{k}$-saturated tree, then $T_{k-1,3} \subseteq T$.

Finally, Kászonyi and Tuza proved that the star has maximum saturation number for trees.

Theorem 4. [KT86] For any tree, $T_{k}$, on $k$ vertices such that $T_{k} \neq S_{k}$, $\operatorname{sat}\left(n, T_{k}\right) \leq$ $\operatorname{sat}\left(n, S_{k}\right)$.

Curiously, we will show that the unique tree on $k$ vertices with smallest saturation number is almost the same graph: a star with a single subdivided edge.

\section{Minimum Saturation Numbers for Trees}

For $k \geq 4$ let $T_{k}^{*}$ be the tree on $k$ vertices obtained by subdividing one edge of a star on $k-1$ vertices. Thus, $T_{k}^{*}$ has a vertex of degree $k-2$ and a vertex of degree 2 with the remaining vertices of degree 1 (See Figure 3 ). First we will show (Lemma 1 ) that $T_{k}^{*}$ is the only tree $T_{k}$ for which there exists a $T_{k}$-saturated tree of order $k$. Then, we will show (Corollary 1) that $\underline{\mathbf{S A T}}\left(n, T_{k}^{*}\right)$ is a set of specific star forests each having $\left\lfloor\frac{n+k-2}{k}\right\rfloor$ components.

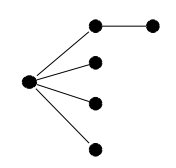

Figure 3: $T_{6}^{*}$

Specifically, let $F$ be the forest on $n$ vertices equal to (1) $(n / k) S_{k}$ if $n \equiv 0 \bmod k$, 
(2) $((n-k-1) / k) S_{k} \cup S_{k+1}$ if $n \equiv 1 \bmod k$,

(3) $((n-k-p) / k) S_{k} \cup K_{2} \cup S_{k+p-2}$ if $n \equiv p \bmod k$ for $2 \leq p \leq k-1$.

Thus, $F$ has $\lfloor(n+k-2) / k\rfloor$ tree components and $n-\lfloor(n+k-2) / k\rfloor$ edges. It is obvious that $F$ does not contain $T_{k}^{*}$ as a subgraph, and it is also clear that the addition of any edge to $F$ will produce a copy of $T_{k}^{*}$. Hence, $F \in \mathbf{S A T}\left(n, T_{k}^{*}\right)$. First we will show that $F \in \underline{\operatorname{SAT}}\left(n, T_{k}^{*}\right)$ and $\boldsymbol{\operatorname { s a t }}\left(n, T_{k}\right)>|E(F)|$ for all $k$-vertex trees $T_{k} \neq T_{k}^{*}$

Lemma 1. If there exists trees $T_{k}$ and $T_{k}^{\prime}$ each of order $k$ such that $T_{k}^{\prime}$ is $T_{k}$-saturated, then $k \geq 4, T_{k}=T_{k}^{*}$, and $T_{k}^{\prime}=S_{k}$.

Proof. When $k=4$, there exist only two trees, $T_{k}^{*}$ and $S_{4}$ and it is easy to see in this case the conclusion holds. For $k=5$, there exist only three trees none of which is $T_{k}$-saturated for any tree on 5 vertices except for $T_{5}^{*}$ and $S_{5}$.

Thus, we assume $k \geq 6$ and that $T_{k}^{\prime}$ is not a star, $S_{k}$. Thus, $T_{k}^{\prime}$ contains a path with at least 4 vertices. Also, since $T_{k}^{\prime}$ is $T_{k}$-saturated and both have order $k$, for every edge $e \notin T_{k}^{\prime}$ there exists an edge $e^{\prime} \in T_{k}^{\prime}$ such that $T_{k}^{\prime}+e-e^{\prime}=T_{k}$. Select a longest path $P$ in $T_{k}^{\prime}$, say $P=\left(x_{1}, x_{2}, \cdots, x_{q-1}, x_{q}\right)$ with $q \geq 4$.

Case 1: Suppose $\operatorname{deg}\left(x_{2}\right) \geq 3$ or $\operatorname{deg}\left(x_{q-1}\right) \geq 3$.

Without loss of generality, assume $\operatorname{deg}\left(x_{2}\right) \geq 3$. Let $y$ be a vertex of degree 1 adjacent to $x_{2}$ other than $x_{1}$. Such a vertex must exist since $\operatorname{deg}\left(x_{2}\right) \geq 3$ and $P$ is a longest path. Let $e=x_{1} y \notin E\left(T_{k}^{\prime}\right)$. Then without loss of generality, $e^{\prime}=y x_{2}$ and we conclude that $T_{k}=T_{k}^{\prime}+e-e^{\prime}$ and, in particular, $T_{k}^{\prime}$ has exactly one more vertex of degree 1 than $T_{k}$. Observe that if $T_{k}^{\prime}$ contains two nonadjacent vertices $u$ and $v$ both of degree 2 or more, then the copy of $T_{k}$ contained in $T_{k}^{\prime}+u v$ would have at least as many vertices of degree 1 as $T_{k}^{\prime}$. Thus every pair of vertices of degree 2 or more in $T_{k}^{\prime}$ is adjacent. This means $T_{k}^{\prime}$ has exactly two vertices of degree 2 or more, say $u$ and $v$. Furthermore, $u$ and $v$ must have the same degree since the copy of $T_{k}$ obtained by adding the edge between neighbors of $u$ must be isomorphic to that obtained by adding the edge between neighbors of $v$. This forces $T_{k}^{\prime}$ to be a symmetric double star.

But now, if we let $e$ be a non-edge between end vertices of a longest path, there is no edge $e^{\prime}$ whose deletion will produce a tree isomorphic to the one obtained when $e$ is between end vertices with a shared neighbor. So $T_{k}^{\prime}$ is not $T_{k}$-saturated for any tree, a contradiction.

Case 2: Suppose $\operatorname{deg}\left(x_{2}\right)=\operatorname{deg}\left(x_{q-1}\right)=2$.

Let $e=x_{1} x_{3} \notin E\left(T_{k}^{\prime}\right)$. Then, in order to avoid a copy of $T_{k}$ in $T_{k}^{\prime}, e^{\prime}=x_{1} x_{2}$. So, $T_{k}=T_{k}^{\prime}+e-e^{\prime}$ and therefore $T_{k}$ must have exactly one more vertex of degree 1 than $T_{k}^{\prime}$. Now consider $T_{k}^{\prime}+x_{1} x_{q}$. To have the right number of vertices of degree 1 in the copy of $T_{k}$, we would have to find an edge in $T_{k}^{\prime}+x_{1} x_{q}$ whose deletion would produce three vertices of degree 1, which is impossible. So, $T_{k}^{\prime}$ cannot contain a path of four or more vertices and is therefore a star. Finally, $T_{k}^{*}$ is the only tree $T_{k}$ for which $T_{k}^{\prime}$ is $T_{k}$-saturated.

Theorem 5. For any tree $T_{k}$ of order $k \geq 5$ and any $n \geq k+2$,

$$
\operatorname{sat}\left(n, T_{k}\right) \geq n-\lfloor(n+k-2) / k\rfloor .
$$

Moreover, $T_{k}^{*}$ is the only tree attaining this minimum for all $n$. 
Proof. Let $G \in \underline{\mathbf{S A T}}\left(n, T_{k}\right)$ for a fixed tree $T_{k}$ of order $k \geq 5$. Observe that any component of $G$ of order less than $k$ must be complete and the union of any pair of components must contain at least $k$ vertices. Since $k \geq 5$, this implies $G$ can have at most one component of the form $K_{i}$ for $i \in\{1,2\}$.

Thus, if $T_{k} \neq T_{k}^{*}$, then Lemma 1 implies that any tree components of $G$ have order at least $k+1$ with the possible exception of a single component of order 2 or less. Thus $\operatorname{sat}\left(n, T_{k}\right)=|E(G)| \geq n-\left\lfloor\frac{n-1}{k+1}\right\rfloor-1 \geq n-\left\lfloor\frac{n+k-2}{k}\right\rfloor$. Furthermore, the previous inequality is strict for $n \geq k^{2}+k+2$.

Assume $T_{k}=T_{k}^{*}$. If $|E(G)|<n-\lfloor(n+k-2) / k\rfloor$, then $G$ has more than $\lfloor(n+k-2) / k\rfloor$ components. Thus, at least two of them have order strictly less than $k$. So they are both complete and together contain at least $k$ vertices. Hence, we could replace these two components with a star on the same number of vertices to create a new graph $G^{\prime}$ that is $T_{k}^{*}$-saturated but with fewer edges, contradicting the assumption $G \in \underline{\mathbf{S A T}}\left(n, T_{k}^{*}\right)$. Hence, $\operatorname{sat}\left(n, T_{k}^{*}\right)=n-\lfloor(n+k-2) / k\rfloor$.

The following corollary follows immediately from the preceding proof.

Corollary 1. For $k \geq 5$, every graph $G \in \underline{\mathbf{S A T}}\left(n, T_{k}^{*}\right)$ is a forest of $\lfloor(n+k-2) / k\rfloor$ stars. If $n-k\lfloor n / k\rfloor \geq 2$, then exactly one of the stars is $K_{2}$.

\section{Subtree Properties}

There are no general monotone properties for subtrees of trees relative to the function $\operatorname{sat}\left(n, T_{k}\right)$. The following theorems verify this in a very strong way.

We introduce some useful notation. Let $G$ be a nonregular graph. Let $x \in V(G)$ such that $\operatorname{deg}(x)>\delta(G)$ and there does not exist a vertex $z \in V(G)$ with $\operatorname{deg}(x)>\operatorname{deg}(z)>$ $\delta(G)$. Define $\delta_{2}(G)=\operatorname{deg}(x)$, that is the second smallest degree in $G$.

Theorem 6. If $T_{k}$ is a tree of order $k \geq 5$ such that $T_{k} \neq S_{k}$ and $\delta_{2}\left(T_{k}\right)=d$, then $\operatorname{sat}\left(n, T_{k}\right) \geq \frac{d-1}{2} n$ provided $n \geq(d-1)^{3}$.

Proof. Let $G \in \underline{\operatorname{SAT}}\left(n, T_{k}\right)$. It is enough to show that $G$ has average degree at least $d-1$. If $\delta(G) \geq d-1$, the result holds so assume $\delta(G) \leq d-2$. Observe that any two vertices of degree $d-2$ or less must be adjacent. Let $x \in V(G)$ such that $d(x)=\delta(G)$. Now all vertices in $V(G) \backslash N[x]$ (where $N[x]$ denotes the closed neighborhood of $x$ ) must have degree at least $d-1$. Furthermore, since $T_{k} \neq S_{k}$, every vertex of $V(G) \backslash N[x]$ must be adjacent to a vertex of degree at least $d$. Thus, $\sum_{v \in G} d(v) \geq \delta(\delta+1)+(n-\delta-1)(d-1)+\frac{n-\delta-1}{d}=$ $n(d-1)+\frac{n}{d}+(\delta+1)^{2}-(\delta+1) \frac{d^{2}+1}{d} \geq n(d-1)$ for $n \geq(d-1)^{3}$ and the result follows.

Corollary 2. For a given tree $T, T$ is the subtree of a tree $T^{\prime}$ such that $\operatorname{sat}\left(n, T^{\prime}\right) \geq \alpha n$ for any constant $\alpha$ and $n$ sufficiently large.

Proof. Let $d=2 \alpha+1$. Construct a tree $T^{\prime}$ such that $\delta_{2}\left(T^{\prime}\right) \geq d$ by adding pendant vertices to those vertices of $T$ with degree 2 or more.

On the other hand we will now show that for a given tree $T, T$ is the subtree of a tree $T^{\prime}$ such that $\operatorname{sat}\left(n, T^{\prime}\right)<n$. We first need to prove a structural lemma. Recall that 
$T_{l, d}$ refers to a perfect $d$-ary tree such that a longest path contains $l$ vertices (See Figure 2.) We will refer to the $d$ subtrees below $r$ (or the $d-1$ subtrees below $r_{i}$ ) to mean the $d$ trees that would result from the deletion of the edges incident to $r$ (or the $d-1$ trees resulting from the deletion of edges incident to $r_{i}$ ). Note that each of these is a standard $(d-1)$-ary tree.

Lemma 2. Given any edge $e \notin E\left(T_{k, d}\right)$, there exists a path in $T_{l, d}+e$ on

(a) $\frac{l+3}{2}$ vertices beginning at $r$ and using vertices from at most two of the subtrees under $r$ for $l$ odd

or

(b) $\left\lceil\frac{l+3}{2}\right\rceil$ vertices ending at one of the roots $r_{i}$ and using vertices from at most one of the $d-1$ subtrees under $r_{i}$ for $l$ even.

Proof. Let $T_{l, d}=T$ be the perfect $d$-ary tree defined above. Assume $l$ is odd. Let $e=y z$ be an edge not in $T$ and assume the level of $y$ is less than or equal to that of $z$.

Case 1: Suppose $z$ lies on the unique $r y$ path $(r=z$ is allowed)

We construct the path on $(l+3) / 2$ vertices as follows. Starting at $r$, take the unique path in $T$ down to $z$, take edge $e=z y$, take the unique path from $y$ up to $z_{c}$, a child of $z$, and from $z_{c}$ take a path down to any end vertex. Recall that $z_{c}$ will have $d-2$ children other than $y$ from which to choose. Since this path includes a path from $r$ down to an end vertex (through $z$ and $z_{c}$ ) and at least one additional vertex, namely $y$, it must contain at least $(l+3) / 2$ vertices. Also, observe that this path uses at most one of the subtrees under $r$, namely the one containing the unique $r y$ path.

Case 2: Suppose $z$ does not lie on the unique ry path

Construct the desired path as follows. Starting at $r$, take the unique path down to $y$, take edge $e=y z$, take any path from $z$ down to an end vertex. Since $z$ is on a level at least as high as $y$, the path contains at least two vertices from the same level and therefore at least $(l+3) / 2$ vertices. Also, observe that it uses at most two subtrees under $r$, namely the one containing $y$ and the one containing $z$ (which may in fact be the same).

Assume $l$ is even. Let $e=y z$ be an edge not in $T$.

Case 1: The edge $e$ lies entirely in the subtree rooted by $r_{1}$ or the subtree rooted by $r_{2}$ Without loss of generality, assume $e$ lies entirely in the tree rooted by $r_{1}$. Then applying the method when $l$ is odd, we know there exists a path on at least $l / 2+1$ vertices starting at $r_{1}$ and completely contained in this subtree. Add edge $r_{1} r_{2}$ and the desired path is obtained using no subtree under $r_{2}$.

Case 2: The edge $e$ contains one vertex from the tree rooted at $r_{1}$ and one from the tree rooted at $r_{2}$

Without loss of generality, assume $y$ is in the subtree rooted at $r_{1}, z$ is in the subtree rooted at $r_{2}$, and that the level of $y$ is no more than that of $z$. Then construct the desired path by starting at $r_{2}$, going down to $z$, taking edge $z y$, take the path from $y$ up to $r_{1}$, and finally take a path from $r_{1}$ down to any end vertex that doesn't require using vertex $y$. Observe that this contains a path from $r_{2}$ to an end vertex under $r_{1}$ plus at least one additional vertex, namely $y$. Thus it must contain at least $l / 2+2$ vertices and it uses vertices from at most one subtree under $r_{2}$, namely the one containing $z$. 
Theorem 7. Let $T$ be a tree with maximum degree $\Delta \geq 3$ and such that a longest path has $l$ vertices. Furthermore, assume that there exists a longest path $P$ in $T$ such that the first $\lceil l / 2\rceil$ vertices on this path have degree 2 or less. Then $T_{l-1, \Delta+1}$ is $T$-saturated and $\operatorname{sat}(n, T)<n$ for $n \geq\left|V\left(T_{l-1, \Delta+1}\right)\right|$.

Proof. Since $T_{l-1, \Delta+1}$ has no path on $l$ vertices, it is $T$-free. Now consider $T_{l-1, \Delta+1}+e$ for some new edge $e$. From Lemma 6 , we know there exists a path, $Q$ on at least $\lceil l / 2\rceil+1$ vertices that ends in a vertex in the top level of $T_{l-1, \Delta+1}$ (either $r$ or $r_{2}$ depending on the parity of $k$ ). Additionally, this top vertex has at least $\Delta-1$ subtrees under it all of which are disjoint from $Q$. Thus, $T_{l-1, \Delta+1}+e$ must contain a copy of $T$.

Let $m=\mid V\left(T_{l-1, \Delta+1} \mid \leq n\right.$. So there exist integers $p$ and $q$ such that $n=p m+q$ such that $0 \leq q>m$. Thus, there exists a $T$-saturated forest consisting of $(p-1)$ copies of

$T_{l-1, \Delta+1}$ and one component formed from a copy of $T_{l-1, \Delta+1}$ with $q$ additional pendant vertices adjacent to vertices in the second level. The upper bound now follows.

Corollary 3. For a given tree $T, T$ is the subtree of a tree $T^{\prime}$ such that $\operatorname{sat}\left(n, T^{\prime}\right)<n$.

Proof. Given tree $T$ with diameter $p$, construct $T^{\prime}$ by adding to $T$ a path on $p+1$ vertices and apply the previous theorem with diameter $m=2 p$.

It should also be observed that the proof of Lemma 2 implies that, for $m$ odd and any new edge $e$, one can find a path on $m$ vertices in $T_{m, d}$ such that the middle vertex (vertex $\lceil m / 2\rceil$ on the path) is in one of the top two levels. Thus, the theorem above can be extended to include trees for which the degree of the middle vertex on a diameter path is greater than 2 provided the longest path starting at this middle vertex away from the diameter path contains at most $(m-3) / 2$ vertices. On the other hand, for $m$ even, by considering an edge $e$ from the top level to the bottom level, we see that we cannot avoid forcing a vertex of degree two close to the middle $(\lfloor m / 2\rfloor)$. Furthermore, by adding the edge from level $r$ to an end vertex directly under it, we see that on every path on $m$ vertices there must be a vertex of degree 2 somewhere in position $m-r+1$ to $m-2 r+4$.

\section{Some Results Concerning Specific Trees}

The following technical lemma simplifies the proof of the saturation number in many cases.

Lemma 3. Assume $T_{k}$ is a tree of order $k \geq 5$ and $T_{l}$ is an $T_{k}$-saturated tree of order $l$ such that

(a) $|V(T)| \geq l$, for every $T_{k}$-saturated tree $T$

(b) for every $m, 1 \leq m \leq l-1$, there exists an $T_{k}$-saturated tree $T_{l+m}$ of order $l+m$, and

(c) the union of any pair of trees in the set $S=\left\{T_{l}, T_{l+1}, T_{l+2}, \cdots T_{2 l-1}\right\}$ is $T_{k}$-saturated. Then for $n \geq l$,

(1) there exists a graph $G \in \underline{\mathbf{S A T}}\left(n, T_{k}\right)$ such that $G$ is a forest and

(2) $n-\left\lfloor\frac{n-1}{l}\right\rfloor-1 \leq \operatorname{sat}\left(n, T_{k}\right) \leq n-\left\lfloor\frac{n}{l}\right\rfloor$. 
Proof. (Part 1) For any $n \geq l$ there exists an $T_{k}$-saturated forest consisting of $\left(\left\lfloor\frac{n}{l}\right\rfloor-\right.$ 1) $T \cup T_{p}$ where $n \equiv p \bmod l$. Call this graph $G^{\prime}$. Let $G \in \underline{\mathbf{S A T}}\left(n, T_{k}\right)$ with the minimum number of components that are not trees. Let $A$ be the set of vertices of $G$ in components that are not trees. Then the graph $G-A=F$ is a (nonempty) forest. Either $F=K_{1}$ or $F=K_{2}$ or $F$ contains a tree on at least $l$ vertices and thus we can assume all such "large" trees are elements from $S$. If $F=K_{1}$ or $F=K_{2}$, then $|E(G)| \geq n-1 \geq\left|E\left(G^{\prime}\right)\right|$. If $F$ contains a large tree, then the vertices of $A$ along with vertices of the large tree can be replaced entirely with elements from $S$, forming an element of $\underline{\mathbf{S A T}}\left(n, T_{k}\right)$ with fewer nontree components.

(Part 2) The upper bound is obtained from the graph $G^{\prime}$ described earlier. The lower bound results from the observation that a minimal $T_{k}$-saturated forest might have $K_{1}$ or $K_{2}$ as a component.

Note that even under the hypotheses of Lemma 3, we do not know that all $G \in$ $\underline{\operatorname{SAT}}\left(n, T_{k}\right)$ are necessarily forests. For example, $T_{3,5} \cup K_{3} \in \underline{\mathbf{S A T}}\left(10, P_{6}\right)$.

\section{Brooms}

First we will consider brooms, denoted $B_{r, k}$, where $r$ corresponds to the number of vertices on the handle and $k$ denotes the number of bristles. So, $B_{r, k}$ contains $r+k$ vertices. (See Figure 4.) The vertex of degree $k+1$ will be referred to as the center of the broom. One of the interesting properties of the collection of all brooms is that it contains all of the trees for which the saturation number is, thus far, known exactly: the star $B_{1, k}$, the path $B_{r, 1}$, and the star with one subdivided edge $B_{3, k}$. In the theorems below, we will find the saturation number for some specific brooms.

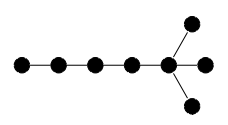

Figure $4: B_{5,3}$

Theorem 8. For $k \geq 2$ and $n \geq 2 k+5$, sat $\left(n, B_{4, k}\right)=n-\left(\left\lfloor\frac{n-2}{2 k+3}\right\rfloor+1\right)$.

Proof. Recall that $S_{a, b}$ is a double star on $a+b$ vertices. See Figure 1. Note $S_{k+2, k+1}$ is $B_{4, k}$-saturated. In addition, $S_{a, b}$ is $B_{4, k}$-saturated for any $a \geq k+2, b \geq k+1$.

In order to apply Lemma 3 , we need to show that every $B_{4, k}$-saturated tree has at least $\left|V\left(S_{k+2, k+1}\right)\right|=2 k+3$ vertices. Let $T$ be any $B_{4, k}$-saturated tree. By adding an edge between two vertices of degree 1 in $T$, we conclude $T$ must have at least one vertex of degree at least $k+1$.

Case 1: Assume $T$ has precisely one vertex of degree at least $k+1$, say $x$.

Then $x$ cannot be adjacent to two vertices of degree 1 since the edge between them would not produce a $B_{4, k}$. Let $y$ be a neighbor of $x$ of degree at least 2 . Then, the length of the longest path in $T$ starting at $x$ and using edge $x y$ is exactly 2. Furthermore, adding the 
edge between $x$ and the end vertex of such a path implies that $\operatorname{deg}(y) \geq 3$. Thus, if $T$ has precisely one vertex of high degree, $|V(T)| \geq 3 k+2 \geq 2 k+3$.

Case 2: Assume $T$ has at least two vertices of degree $k+1$ or more.

Then, to avoid a $B_{4, k}$, these vertices must be adjacent. Thus, there are precisely two vertices of high degree, say $u$ and $v$, and all neighbors of $u$ and $v$ (other than $u$ and $v$ ) are end vertices. Thus, by adding the edge between $x \in N(u)-v$ and $y \in N(v)-u$, we conclude at least one of $u$ or $v$ must have degree at least $k+2$. So, every nontrivial tree component $T$ must have at least $2 k+3$ vertices.

Finally, $S_{k+2, k+1} \cup K_{2}$ is $B_{4, k}$-saturated. So, $\operatorname{sat}\left(n, B_{4, k}\right) \leq n-\left(\left\lfloor\frac{n-2}{2 k+3}\right\rfloor+1\right)$. But, by the argument above, there does not exist any tree $T$ such that $T \cup K_{1}$ is $B_{4, k}$-saturated. So, by Lemma $3, \operatorname{sat}\left(n, B_{4, k}\right) \geq n-\left(\left\lfloor\frac{n-2}{2 k+3}\right\rfloor+1\right)$. Thus, for $n \geq 2 k+5$, the graph consisting of a disjoint union of one $K_{2}$ and $\left\lfloor\frac{n-2}{2 k+3}\right\rfloor$ double stars each of which has $S_{k+2, k+1}$ as a subgraph is a minimal $B_{4, k}$-saturated graph and $\operatorname{sat}\left(n, B_{4, k}\right)=n-\left(\left\lfloor\frac{n-2}{2 k+3}\right\rfloor+1\right)$.

Theorem 9. For $k \geq 2$ and $n \geq 2 k+6$, $\operatorname{sat}\left(n, B_{5, k}\right)=n-\left\lfloor\frac{n}{2 k+6}\right\rfloor$.

Proof. Recall that $T_{5,3}$ is the perfect 3 -ary tree such that a longest path has 5 vertices and it is $P_{6}$-saturated. (See Figure 2.) Let $u$ and $v$ be any two of the three vertices of $T_{5,3}$ in the middle level (those adjacent to vertices of degree 1.) Define $T_{5,3}^{k}$ to be the tree constructed from $T_{5,3}$ by adding an additional $k-2$ pendant vertices to each of $u$ and $v$. (See Figure 5.) Note $T_{5,3}^{k}$ has two vertices of degree $k+1$ and is still $P_{6}$-saturated. It is easy to check that for every new edge $e$, the graph $T_{5,3}^{k}+e$ contains a $P_{6}=v_{1} v_{2} \cdots v_{6}$ such that $\operatorname{deg}_{T_{5,3}^{k}}\left(v_{2}\right)=k+1$. Thus $T_{5,3}^{k}$ and $T_{5,3}^{k} \cup T_{5,3}^{k}$ are $B_{5, k}$-saturated. Furthermore, any number of additional pendant vertices can be added to any of the vertices in the middle level of $T_{5,3}^{k}$ and the resulting graph will still be $B_{5, k}$-saturated.

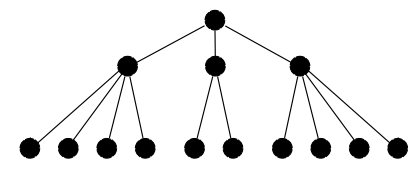

Figure 5: $T_{5,3}^{4}$

Next we show that if $T$ is a $B_{5, k}$-saturated tree, then $|V(T)| \geq 2 k+6$. By adding the edge between any two vertices of degree 1 , we see $T$ must contain a vertex of degree at least $k+1$.

Case 1: Assume $T$ has precisely one vertex of degree at least $k+1$, say $x$.

At most one of its neighbors can have degree 1 . Thus, let $y$ be a neighbor of $x$ of degree at least 2. If the longest path starting at $x$ and proceeding through $y$ is of length 2 , then adding the edge from $x$ to the end vertex of this path cannot produce a $B_{5, k}$. Thus, the longest path in $T$ starting at $x$ and using edge $x y$ has exactly 4 vertices. Furthermore, 
adding the edge between $x$ and the end vertex of such a path implies that the component of $T-x$ containing $y$ has at least 4 vertices. Thus, if $T$ has precisely one vertex of high degree, $|V(T)| \geq 4 k+2 \geq 2 k+6$.

Case 2: Assume $T$ has at least two vertices of degree $k+1$ or more.

Find two high degree vertices farthest apart, say $x_{1}$ and $x_{2}$. Then $d\left(x_{1}, x_{2}\right) \leq 2$. If $x_{1} x_{2} \in E(T)$, then neither can be adjacent to two vertices of degree 1 . Let $y$ be a neighbor of $x_{1}$ of degree at least $2\left(y \neq x_{2}\right.$.) Adding the edge between $x$ and an end vertex of a longest path through $y$ implies $\operatorname{deg}(y) \geq 3$. Applying symmetry, we find $|V(T)| \geq\left|\left\{x_{1}, x_{2}\right\}\right|+2\left(3\left|N\left(x_{1}\right)\right|-2\right)=6 k-2 \geq 2 k+6$. If $d\left(x_{1}, x_{2}\right)=2$, then label as $y$ the vertex on the path between them. Now all neighbors of $x_{i}$ other than $y$ must have degree 1. The copy of $B_{5, k}$ in $T+\left\{x_{1} x_{2}\right\}$ requires $T$ to have a path on three vertices starting at $y$ and disjoint from $x_{1}$ and $x_{2}$. Call it $y y_{1} y_{2}$. Now $\operatorname{deg}\left(y_{2}\right)=1$. Adding the edge $y y_{2}$ forces $\operatorname{deg}\left(y_{1}\right) \geq 3$. Thus, $|V(T)| \geq 2 k+6$.

Thus, we have shown that all $B_{5, k}$-saturated trees have at least $2 k+6$ vertices.

Finally, observe that for any $B_{5, k}$-saturated tree $T$, the graph $T \cup K_{i}$ is not $B_{5, k^{-}}$ saturated for $i=1,2$ since adding an edge between $K_{i}$ and vertex of degree $k+1$ in $T$ cannot produce a copy of $B_{5, k}$. Thus, by applying Lemma 3 we know that $\underline{\operatorname{SAT}}\left(n, B_{5, k}\right)$ contains a forest each component of which has $T_{5,3}^{k}$ as a subgraph and $\operatorname{sat}\left(n, B_{5, k}\right)=$ $n-\left\lfloor\frac{n}{2 k+6}\right\rfloor$.

Theorem 10. For $n \geq a_{r+1}$ and $r \geq 5$, then $\operatorname{sat}\left(n, B_{r, 2}\right)=\operatorname{sat}\left(n, P_{r+1}\right)$ where

$a_{r+1}= \begin{cases}2^{m+1}-2 & \text { if } r=2 m, \\ 3 \cdot 2^{m}-2 & \text { if } r=2 m+1 .\end{cases}$

Proof. Recall that if $G \in \underline{\mathbf{S A T}}\left(n, P_{r+1}\right)$, then $G$ is a forest such that all of its components contain a common minimal subtree on $a_{r+1}$ vertices called a perfect 3 -ary tree and labeled $T_{r, 3}$. It is easy to verify that these graphs are also $B_{r, 2}$-saturated. Thus, $\operatorname{sat}\left(n, B_{r, 2}\right) \leq$ $\operatorname{sat}\left(n, P_{r+1}\right)$.

Now, we need to show that every $B_{r, 2}$-saturated tree $T$ contains at least $a_{r+1}$ vertices. We will do this by showing that every $B_{r, 2}$-saturated tree must be $P_{r+1}$-saturated.

Assume there exists a $B_{r, 2}$-saturated graph, $T$, that contains a path on $r+1$ vertices. Let $P=x_{1}, x_{2}, \cdots, x_{s}$ be a longest path in $T$. So $s \geq r+1$. Since $P$ is not itself $B_{r, 2^{-}}$ saturated, $P$ must contain a vertex of degree at least 3 . Let $x_{i_{0}}$ be the first vertex on $P$ of degree 3 or more. Then $3 \leq i_{0} \leq r-1$. Let $e=x_{1} x_{i_{0}}$ and let $c$ be the vertex of $T$ that is the center of the copy of $B_{r, 2}$ in $T+e$. (That is, $\operatorname{deg}_{B_{r, 2}}(c)=3$.) We know $c \neq x_{i}$ for $1 \leq i \leq i_{0}-1$ because all these vertices have degree 2 in $T+e$. But for every other choice of $c$, the edge $e$ must appear in a path on $r$ vertices starting at $c$ which immediately implies $B_{r, 2} \subset T$, a contradiction. So the longest path in any $B_{r, 2}$-saturated tree, $T$, is at most $r$. Thus, $T$ is $P_{r+1}$ saturated and therefore contains at least $a_{r+1}$ vertices.

\section{Double Stars}

Theorem 11. For $n \geq 6, \operatorname{sat}\left(n, S_{3,3}\right)= \begin{cases}n & n \equiv 0 \bmod 3, \\ n+1 & \text { otherwise. }\end{cases}$ 
Proof. If $n \equiv 0 \bmod 3$, then the graph consisting of $n / 3$ disjoint triangles provides an upper bound for $\operatorname{sat}\left(n, S_{3,3}\right)$. If $n \equiv 1 \bmod 3$, the graph consisting of the union of $(n-7) / 3$ disjoint triangles and one component on 7 vertices consisting of two triangles connected by a single path of length 2 provides an upper bound for $\operatorname{sat}\left(n, S_{3,3}\right)$. The example in the case that $n \equiv 2 \bmod 3$ consists of the union of $(n-8) / 3$ disjoint triangles and one component on 8 vertices consisting of a 4 -cycle and a 5 -cycle which share a single vertex.

Let $G \in \underline{\mathbf{S A T}}\left(n, S_{3,3}\right)$. We want to show that $|E(G)| \geq n$ or $n+1$ accordingly. First, observe that $G$ cannot have two nonadjacent vertices of degree 1 since adding the edge between them cannot produce a copy of $S_{3,3}$. Also, if a component of $G$ is a cycle, it must be a 3 -cycle since larger cycles are not $S_{3,3}$-saturated. Thus, if $\delta(G) \geq 2$ the result follows.

Assume $G$ contains a vertex $x$ such that $\operatorname{deg}(x) \leq 1$.

Case 1: The vertex $x$ is in a component of order at most 2 .

Let $C$ and $C^{\prime}$ be distinct components of $G$ such that $x \in C$. Note, it is enough to show that the average degree of vertices in $G$ is strictly greater than 2. By adding edges from $x$ to vertices in $C^{\prime}$, it follows that $\delta\left(C^{\prime}\right) \geq 2$, and $C^{\prime}$ must have at least two vertices of degree 3 or more that are adjacent and that share a common neighbor. If $G$ has three or more components, the result follows. If $G=C \cup C^{\prime}$, the average degree of $G$ will be above 2 unless $C^{\prime}$ has exactly two vertices of degree 3 and all others have degree 2 . This forces $C^{\prime}$ to be a cycle with a single chord or two cycles connected by a path. In neither case is the graph $S_{3,3}$-saturated.

Case 2: The vertex $x$ is in a component of order at least 3 .

Then $x$ is in a component of order at least 6 . It is enough to show that the average degree in this component is more than 2 . Note that the only connected graph with average degree exactly 2 and precisely one vertex of degree 1 is a cycle with a pendant path. This is not $S_{3,3}$-saturated.

Theorem 12. For $n \geq(t+1)^{3}, t n / 2 \leq \operatorname{sat}\left(n, S_{t+1, t+1}\right) \leq \frac{t n}{2}+\frac{t(t+2)}{2}$.

Proof. Let $q=\lfloor n /(t+1)\rfloor$ and $r=n-q(t+1)$. Then the graph $G=(q-r) K_{t+1} \cup r K_{t+2}$ is $S_{t+1, t+1}$-saturated and $|E(G)| \leq \frac{t(n-r)}{2}+r(t+2) \leq \frac{t n}{2}+\frac{t(t+1)}{2}$.

For the lower bound, apply Theorem 6.

Theorem 13. Given the double star $S_{t+1, s+1}$ where $t>s$ and $n \geq(s+1)^{3}$, then $\left(\frac{s}{2}\right) n \leq$ $\operatorname{sat}\left(n, S_{t+1, s+1}\right) \leq \frac{s+1}{2} n-\frac{s^{2}+8}{8}$.

Proof. To obtain the upper bound, consider the graph $G=K_{1}+H$ where $H \in \underline{\mathbf{S A T}}(n-$ $\left.1, S_{s+1}\right)$. The graph $G$ is $S_{t+1, s+1}$-saturated and $|E(G)|=n-1+\frac{s-1}{2} n-\frac{s^{2}}{8}=\frac{s+1}{2} n-\frac{s^{2}+8}{8}$.

To obtain the lower bound, apply Theorem 6 .

\section{Subdivided Stars}

Let $S_{r+1}^{t}$ denote the graph obtain by subdividing $t$ edges of a star on $r+1$ vertices. Hence, $t \leq r$. (See Figure 6.)

So $S_{r+1}^{t}$ contains $t+r+1$ vertices, of which $r$ have degree $1, t$ have degree 2 , and one has degree $r$. The vertex of degree $r$ is called the center of the subdivided star. Recall 


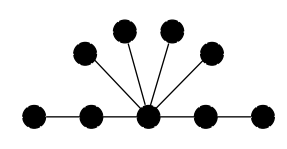

Figure 6: $S_{7}^{2}$

that the tree on $k$ vertices of minimum saturation number (previously referred to as $T_{k}^{*}$ ) can be thought of as $S_{k-1}^{1}$.

We will find exact values for $\operatorname{sat}\left(n, S_{r+1}^{2}\right)$ for all $r$ and establish upper and lower bounds for $\operatorname{sat}\left(n, S_{r+1}^{t}\right)$ for $t \geq 3$.

Theorem 14. For $n \geq 10$, $\operatorname{sat}\left(n, S_{4}^{2}\right)=\left\lceil\frac{9 n}{10}\right\rceil$.

Proof. Let $P_{6}=x_{1} x_{2} \cdots x_{6}$ be a path on 6 vertices. Let $H$ be the graph on 10 vertices constructed from $P_{6}$ by adding precisely two pendant vertices to each of $x_{2}$ and $x_{5}$. Then the forest $G=\left(\left\lfloor\frac{n}{10}\right\rfloor-1\right) H \cup H^{*}$ where $H^{*}$ is a copy of $H$ with $s \equiv n \bmod 10$ additional pendant vertices added to $x_{2}$. Then $G$ is $S_{4}^{2}$-saturated.

We need to show that $H$ is an $S_{4}^{2}$-saturated tree of smallest order. Let $T$ be any $S_{4}^{2}$-saturated tree and let $P=x_{1} x_{2} \cdots x_{m}$ be a longest path in $T$. Since neither a star nor a double star can be $S_{4}^{2}$-saturated, we know $m \geq 5$. In $T+x_{1} x_{3}$, vertex $x_{3}$ must be the center of the newly obtained copy of $S_{4}^{2}$ and vertex $x_{2}$ must be adjacent to an additional pendant vertex. Adding the edge between these two vertices of degree 1 , forces vertex $x_{2}$ to have three pendant vertices. But this argument applies at the other end of the longest path. It is simple to check that if $m=5$ the graph is not $S_{4}^{2}$-saturated. Thus, $m=6$ and $H$ is the minimal tree. Observe that neither $K_{1}$ nor $K_{2}$ can be components and the result follows.

Theorem 15. If $r \geq 4$ and $n \geq r^{2}-r+1$, then $\operatorname{sat}\left(n, S_{r+1}^{2}\right)=n-\left\lfloor\frac{n+r-1}{r^{2}}\right\rfloor$.

Proof. Let $H$ be a rooted tree with three levels such that the root $v$ has $r-1$ neighbors and each of these has $r$ children. Observe that $H$ is $S_{r+1}^{2}$-saturated as is $H \cup H$ and any graph constructed by adding additional pendant vertices to those vertices in the middle level. Let $H^{*}$ be the tree constructed from $H$ by deleting $r-1$ pendant vertices from a single vertex. Thus, in $H^{*}$ all vertices in the second level have $r$ children except for one which has only one child. Note $H^{*}$ is $S_{r+1}^{2}$-saturated as is any graph obtained from $H^{*}$ by adding pendant vertices to those with $r$ children. Note, $H^{*} \cup H^{*}$ is not $S_{r+1}^{2}$-saturated. In fact no $S_{r+1}^{2}$-saturated graph can have two nonadjacent vertices each of degree 2 . Thus, given any $n \geq r^{2}-r+1$, there exists an $S_{r+1^{-}}^{2}$ saturated forest each component of which is a copy of $H^{*}$, a copy $H$, a copy of $H^{*}$ with some added pendant vertices, or a copy of $H$ with some added pen! dant vertices.

We will now show that $H^{*}$ is the smallest $S_{r+1}^{2}$-saturated tree and if $T$ is a tree such that $H^{*} \cup T$ is $S_{r+1}^{2}$-saturated, then $|V(T)| \geq|V(H)|$. Let $T$ be an $S_{r+1}^{2}$-saturated tree. Since neither the star nor the double star are $S_{r+1}^{2}$-saturated, we know the longest path in $T$ has at least 5 vertices. If there exists a vertex $x$ adjacent to two pendant vertices, then $x$ must be the center of the copy of $S_{r+1}^{2}$ obtained by adding the edge between these 
end vertices. Thus, $x$ must be adjacent to at least $r$ pendant vertices. So, any vertex adjacent to a pendant vertex is adjacent to exactly one pendant vertex or at least $r$ pendant vertices. Thus, without loss of generality, if the the longest path in $T$ is labeled $x_{1} x_{2} \cdots x_{m}$, we can assume $x_{2}$ is adjacent to at least $r$ vertices of degree 1 . Now $x_{3}$ must be the center of the copy of $S_{r+1}^{2}$ formed by the addition of edge $x_{1} x_{3}$. Thus, $x_{3}$ has exactly $r-3$ ne! ighbors off the path. If any one of the neighbors of $x_{3}$ is a pendant vertex, adding the edge between it and vertex $x_{2}$ will produce a contradiction. Finally, at most one of the $r-1$ neighbors of $x_{3}$ can have degree 2 . Thus all but at most one must be adjacent to at least $r$ pendant vertices. Thus all $S_{r+1}^{2}$-saturated trees have at least $\left|V\left(H^{*}\right)\right|$ vertices and any longest path in such a tree shares the structure of $H^{*}$ at one end. Since no $S_{r+1}^{2}$-saturated graph can have two nonadjacent vertices of degree two, the result follows.

Next we will find upper and lower bounds for $\operatorname{sat}\left(n, S_{r+1, t}\right)$ when $t \geq 3$. Note that one consequence of the following theorem is that there exist trees with many vertices of degree 2 (for example when $t=r$ ) with "high" saturation number.

Theorem 16. For $r \geq t \geq 3$ and $n \geq 3 t, n \leq \operatorname{sat}\left(n, S_{r+1}^{t}\right) \leq n+3 t-5$

Proof. The graph $G=\left\{K_{1}+\left\{(t-1) K_{3} \cup(n-3 t+2) K_{1}\right\}\right\} \cup K_{2}$ is $S_{r+1}^{t}$-saturated and proves the upper bound.

For the lower bound we argue by contradiction first that no nontrivial component of an $S_{r+1}^{t}$-saturated graph can be a tree and second, that no nontrivial component is unicyclic. Assume that there exists a tree $T$ that is $S_{r+1}^{t}$-saturated. Now find a longest path, $P=x_{1}, x_{2}, \cdots, x_{k}$ in $T$. Then $k \geq 5$. If $x_{2}$ is adjacent to two end vertices, then adding the edge between them forces $x_{2}$ to be the center of a copy of $S_{r+1}^{t}$ which would force $T$ to contain a path longer than $P$. Thus, $\operatorname{deg}\left(x_{2}\right)=2$. Now consider $T+\left\{x_{1} x_{3}\right\}$. The vertex $x_{3}$ must be the center of the newly obtained copy of $S_{r+1}^{t}$ and the new edge must be used as a pendant edge in $S_{r+1}^{t}$. Thus, if $t=r, S_{r+1}^{r}$ has no pendant edges and a contradiction occurs. If $t<r, \operatorname{deg}\left(x_{3}\right)=r-1$ and $T$ must contain exactly $t-1$ additional paths of length exactly 2 starting at $x_{3}$ disjoint from the path $P$. For each of these paths the vertex adjacent to $x_{3}$ has degree exactly 2 . Label one such vertex $y$. Now $T+\left\{x_{2} y\right\}$ fails to contain a copy of $S_{r+1}^{t}$. So there does not exist an $S_{r+1}^{t}$-saturated tree.

Assume there exists a unicyclic $S_{r+1}^{t}$-saturated graph $G$. Clearly, $G$ cannot simply be a cycle, so $G$ is a cycle with pendant trees. Let $x$ be a vertex whose distance from the cycle is maximized. If $x$ is a distance 1 away from the cycle, let $y$ be the neighbor of $x$ and let $y^{-}$be a neighbor of $y$ on the cycle. Then $G+\left\{x y^{-}\right\}$fails to produce a copy of $S_{r+1}^{t}$. So $x$ is at least a distance 2 away. Now the argument used on trees will show no such graph is $S_{r+1}^{t}$-saturated. Thus, if $G$ is a nontrivial $S_{r+1}^{t}$-saturated graph, then $|E(G)| \geq|V(G)|+1$ and the lower bound follows.

\section{Caterpillars}

Define a caterpillar, $P_{k}^{d}$, to be a path on $k \geq 5$ vertices such that all interior ver- 
tices of the path are adjacent to $d$ additional pendant vertices. (See Figure 7.) Thus, $\left|V\left(P_{k}^{d}\right)\right|=k+(k-2) d$. Note that if $k=4$, the graph would be a double star. If $k=3$, the graph is a star.

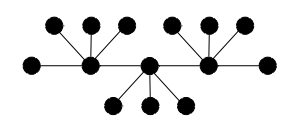

Figure 7: $P_{5}^{3}$

Theorem 17. If $n \geq 2 k-2$ and $k \geq 5$, then

$$
n \leq \operatorname{sat}\left(n, P_{k}^{1}\right) \leq(k-3)\left(\frac{k-2}{2}\left\lfloor\frac{n}{2 k-2}\right\rfloor\right) .
$$

Proof. Let $H=K_{k-3}+\bar{K}_{k+1}$. Let $G=\left(\left\lfloor\frac{n}{2 k-2}\right\rfloor-1\right) H \cup\left(K_{k-3}+\bar{K}_{k+r+1}\right)$ where $r \equiv n$ $\bmod 2 k-2$. Note $G$ is $P_{k}^{1}$-saturated. The lower bound follows from the observation that if a $P_{k}^{1}$-saturated graph has a vertex $x$ of degree 0 (or 1 ), then all $n-1$ (or $n-2$ ) vertices nonadjacent to $x$ have degree at least 2. Furthermore at least 3 vertices have degree 3 or more.

Theorem 18. For $n \geq k+(k-2) d, k \geq 4$ and $d \geq 2, \frac{d+1}{2} n-\frac{(d+2)^{2}}{8} \leq \operatorname{sat}\left(n, P_{k}^{d}\right) \leq$ $\frac{2 k+d-7}{2} n-\frac{(k-3)(k-2)}{2}-\frac{d^{2}}{8}$.

Proof. The lower bound follows directly from Theorem 6 and the fact that $\delta_{2}\left(P_{k}^{d}\right)=d+2$. The upper bound follows from the observation $G=K_{k-3}+H$ where $H \in \underline{\mathbf{S A T}}(n-k+$ $\left.3, S_{d+1}\right)$ is $P_{k}^{d}$-saturated.

Assume that, given a $P_{k}^{d}$, we lengthen the path on $k$ vertices by adding a vertex at each end (see Figure 8.) This appended caterpillar (call it $A P_{k+2}^{d}$ ) has a path on $k+2$ vertices such that the middle $k-4$ vertices have degree $d+2$ and it has two vertices of degree 2 .

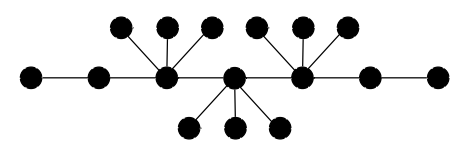

Figure 8: $A P_{7}^{3}$

Theorem 19. For the graph $A P_{k}^{d}, n \geq k+(k-4) d, k \geq 6$ and $d \geq 2$, we have $n \leq$ $\operatorname{sat}\left(n, A P_{k}^{d}\right) \leq n(k-3)+(k-3)\left\lfloor\frac{n}{k+(k-4) d}\right\rfloor((k-4) d+2)$. 
Proof. Let $H=K_{k-3}+\bar{K}_{(k-4) d+3}$. Then the graph $G=\left(\left\lfloor\frac{n}{k+(k-4) d}\right\rfloor-1\right) H \cup\left(K_{k-3}+\right.$ $\left.\bar{K}_{(k-4) d+3+r}\right)$ where $r \equiv n \bmod k+(k-4) d$ is $A P_{k}^{d}$-saturated.

The lower bound can be obtained by observing no nontrivial component of an $A P_{k}^{d}$ saturated graph can be a tree or unicyclic. The longest path argument used in the proofs in the section on subdivided stars will apply.

On the other hand, we know that if we append to $A P_{k}^{d}$ a path on $k$ vertices at one end to make a sort of "one-sided" caterpillar, then the saturation number drops below $n$.

\section{Collections of Paths}

As a last example, we consider a forest such that each component is a $P_{3}$. In fact $\operatorname{both} \operatorname{sat}\left(n, t P_{2}\right)$ and $\underline{\mathbf{S A T}}\left(n, t P_{2}\right)$ are established in [KT86] and [Mad73]. Additionally, $\operatorname{sat}\left(n, P_{3}\right)$ is shown in Theorem 1.

Theorem 20. For $n \geq 6$, $\operatorname{sat}\left(n, 2 P_{3}\right)=\left\lfloor\frac{n+6}{2}\right\rfloor$.

For $t \geq 3$ and $n \geq 6 t-6,\left\lfloor\frac{n+3 t+1}{2}\right\rfloor \leq \operatorname{sat}\left(n, t P_{3}\right) \leq\left\lfloor\frac{n+6(t-1)}{2}\right\rfloor$.

Proof. The graph $N$ (sometimes called a net) consists of a $K_{3}$ such that each vertex of this triangle is adjacent to precisely one pendant vertex. So $N$ has 6 vertices and 6 edges. The graph $H_{n, t}$ is the graph on $n$ vertices consisting of $(t-1)$ copies of $N$ and $\left\lfloor\frac{n-6(t-1)}{2}\right\rfloor$ copies of $K_{2}$. If $n$ is odd, one component of $H_{n, t}$ is an isolated vertex. Then $H_{n, t}$ is $t P_{3}$-saturated for all $t \geq 2$.

Assume $t \geq 2$. Let $G \in \underline{\mathbf{S A T}}\left(n, t P_{3}\right)$. Then $G$ can have at most one isolated vertex. Also, if $G$ contains a vertex $v$ of degree 2 such that its neighbors are not adjacent, the new copy of $P_{3}$ obtained by adding the edge between neighbors of $v$ implies an unused copy of $P_{3}$ in $G$ disjoint for the other $t-1$ copies of $P_{3}$. Thus, the neighbors of vertices of degree 2 must be adjacent. The same argument shows that no vertex can be adjacent to two vertices of degree 1 . Thus, there does not exist a $t P_{3}$ saturated tree for $t \geq 2$. The lower bound for $\operatorname{sat}\left(n, 2 P_{3}\right)$ now follows.

Assume $3 \leq r \leq t$ and $C$ is a nontrivial component of a $r P_{3}$ saturated graph. Since $C$ is not a tree it contains at least one cycle. However, if $C$ is unicyclic, then the previous observations imply that $C$ consists of a cycle such that every vertex is adjacent to exactly one vertex of degree 1 and such a graph is not $r P_{3}$ saturated. Thus, $C$ must contain at least 2 cycles. Thus a minimum of edges would be achieved by a single $t P_{3}$-saturated component and the remaining vertices in a matching. The number of edges in this graph is at least $3 t+1+\left\lfloor\frac{n-3 t}{2}\right\rfloor$.

\section{A Summary of Small Order Cases}

By applying some of the previous theorems and checking a few specific cases, we can establish the saturation numbers for all the trees of order 7 or less. Some of the special cases (denoted by $*$ ) required extensive case analysis which we do not include here. For a fixed number of vertices, the trees are listed in order of increasing saturation number. See Table 1 for trees of order 6 or less and Table 2 for trees of order seven. 


\begin{tabular}{|c|c|c|c|c|}
\hline Order & $\overline{\text { Tree }}$ & & $\overline{\text { sat }(T, n)}$ & Ref. \\
\hline$\overline{2}$ & $\because$ & 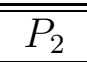 & 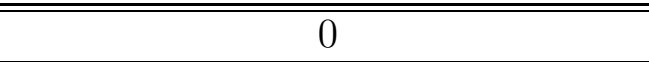 & $\overline{[\mathrm{KT} 86]}$ \\
\hline 3 & $\because \bullet$ & $P_{3}$ & {$[n / 2\rfloor$} & [KT86] \\
\hline 4 & $\bullet \bullet \bullet \bullet$ & $P_{4}$ & $n / 2$ for $n$ even; $(n+3) / 2$ for $n$ odd & [KT86] \\
\hline 4 & & $S_{4}$ & $n-1$ & {$[\mathrm{KT} 86]$} \\
\hline 5 & $\bullet \bullet$ & $B_{3,2}$ & $\lceil(4 n-3) / 5\rceil$ & Cor 1 \\
\hline 5 & $\bullet \bullet \bullet \bullet \bullet$ & $P_{5}$ & $\overline{\lceil(5 n-4) / 6\rceil}$ & [KT86] \\
\hline 5 & & $S_{5}$ & $\lceil(3 n-6) / 2\rceil$ & {$[\mathrm{KT} 86]$} \\
\hline 6 & & $B_{3,3}$ & $\lceil(5 n-4) / 6\rceil$ & Cor 1 \\
\hline 6 & $\ldots \ldots$ & $B_{4,2}$ & $\lceil(6 n-5) / 7\rceil$ & Thm 8 \\
\hline 6 & $\because \ldots$ & $S_{4}^{2}$ & $\lceil 9 n / 10\rceil$ & Thm 14 \\
\hline 6 & $\because \because \bullet \bullet$ & $P_{6}$ & $\lceil 9 n / 10\rceil$ & {$[\mathrm{KT} 86]$} \\
\hline 6 & $\therefore 6$ & $S_{3,3}$ & $n$ for $n \equiv_{3} 0 ; n+1$ otherwise & Thm 11 \\
\hline 6 & 10 & $S_{6}$ & $2 n-3$ & [KT86] \\
\hline
\end{tabular}

Table 1: Saturation Numbers for Trees of Order 6 or Less

\section{Concluding Remarks}

In conclusion, it is fair to say that what we don't know about the saturation number of trees is somewhat larger than what we do know. Specifically, if for $T$ a tree we let $k=|V(T)|$ be fixed, we know the maximum and minimum values of $\operatorname{sat}(n, T)$. For some specific trees, we know sat $(n, T)$ exactly. These include paths and stars, and some brooms, double stars, and subdivided stars. It is interesting that of all the trees for which the exact saturation is known, all but one $\left(S_{4}^{3}\right)$ have a similar structure: the existence of a dominating path. We have upper and lower bounds (some better than others) for other classes of trees including all double stars and some subdivided stars and caterpillars. Finally, we do know of certain properties in trees that effect the saturation number. Specifically, trees with long induced paths will have saturation numbers near the minimum and trees with high values for $\delta_{2}(T)$ will have high saturation numbers.

Obviously, we do not know the saturation number of an arbitrary tree. Additionally, assuming the tree $T$ does not have a high $\delta_{2}$ value and does not have a long induced path, we really don't have any tool other than the Kászonyi-Tuza upper bound result to even put bounds on $\operatorname{sat}(n, T)$. Some more specific open questions follow.

Question 1: Among all trees of order $k$, can you determine the tree of second highest and second lowest saturation number? 


\begin{tabular}{|c|c|c|c|c|}
\hline Order & Tree & & $\operatorname{sat}(T, n)$ & Ref. \\
\hline 7 & & $B_{3,4}$ & $\lceil(6 n-5) / 7\rceil$ & Cor 1 \\
\hline 7 & $\bullet \bullet$ & $B_{4,3}$ & $\lceil(8 n-7) / 9\rceil$ & Thm 8 \\
\hline 7 & $\bullet-$ & $B_{5,2}$ & $\lceil 9 n / 10\rceil$ & Thm 9 \\
\hline 7 & $\cdots \cdots \cdots$ & $C_{6}(0,1,0,0)$ & $\lceil 9 n / 10\rceil$ & $*$ \\
\hline 7 & $\bullet \bullet-\bullet \bullet \bullet \bullet \bullet \bullet$ & $P_{7}$ & $\lceil 13 n / 14\rceil$ & [KT86] \\
\hline 7 & & $S_{5}^{2}$ & $\lceil(15 n-3) / 16\rceil$ & Thm 15 \\
\hline 7 & & $C_{5}(1,1,0)$ & $n+1$ & $*$ \\
\hline 7 & & $S_{4}^{3}$ & $n+3$ & Thm 16 \\
\hline 7 & $\therefore \ldots$ & $C_{5}(1,0,1)$ & $\lfloor(7 n-3) / 6\rfloor$ & $*$ \\
\hline 7 & & $S_{3,4}$ & $\lceil(5 n-3) / 4\rceil$ & Thm 13 \\
\hline 7 & & $S_{7}$ & $\lceil(5 n-9) / 2\rceil$ & [KT86] \\
\hline
\end{tabular}

Table 2: Saturation Numbers for Trees of Order 7

Question 2: Can sharper bounds be established for Theorems 12, 13, 16-19?

Question 3: For those classes of trees for which the saturation number is known, is it possible to characterize the set of minimal saturated graphs?

\section{References}

[Bol67] B. Bollobás. On a conjecture of Erdős, Hajnal and Moon. Amer. Math. Monthly, 74:178-179, 1967.

[CFG08] Guantao Chen, Ralph J. Faudree, and Ronald J. Gould. Saturation numbers of books. Electron. J. Combin., 15(1):Research Paper 118, 12, 2008.

[Che09] Ya-Chen Chen. Minimum c5-saturated graphs. J. Graph Theory, 61, 2009.

[CL05] G. Chartrand and L. Lesniak. Graphs \& digraphs. Chapman \& Hall/CRC, Boca Raton, FL, fourth edition, 2005.

[EHM64] P. Erdős, A. Hajnal, and J. W. Moon. A problem in graph theory. Amer. Math. Monthly, 71:1107-1110, 1964.

[FFS09] J. Faudree, R. Faudree, and J. Schmitt. Survey of graph saturation numbers. preprint, 2009. 
[Für09] Zoltán Füredi. personal communication. 2009.

[GGL95] R. L. Graham, M. Grötschel, and L. Lovász, editors. Handbook of combinatorics. Vol. 1, 2. Elsevier Science B.V., Amsterdam, 1995.

[KT86] L. Kászonyi and Zs. Tuza. Saturated graphs with minimal number of edges. $J$. Graph Theory, 10(2):203-210, 1986.

[Mad73] W. Mader. 1-faktoren in graphen. Math. Ann., 201:269-282, 1973.

[Oll72] L. Taylor Ollmann. $K_{2,2}$ saturated graphs with a minimal number of edges. In Proceedings of the Third Southeastern Conference on Combinatorics, Graph Theory, and Computing (Florida Atlantic Univ., Boca Raton, Fla., 1972), pages 367-392, Boca Raton, Fla., 1972. Florida Atlantic Univ.

[Pik04] Oleg Pikhurko. Results and open problems on minimum saturated hypergraphs. Ars Combin., 72:111-127, 2004.

[Tur41] Paul Turán. Eine Extremalaufgabe aus der Graphentheorie. Mat. Fiz. Lapok, 48:436-452, 1941. 